\title{
Basal cell carcinoma of the eyelids and solar ultraviolet radiation exposure
}

\author{
Gun Lindgren, Brian L Diffey, Olle Larkö
}

\begin{abstract}
Aims-To compare the distribution of eyelid basal cell carcinoma (BCC) with the relative ultraviolet radiation (UVR) exposure to different sites on the eyelids. Methods-The location of BCC on the eyelids was allocated to one of seven regions. The UVR exposure was recorded with a polymer film attached to the eyelids at seven sites in a manikin and in human subjects.

Results-Localisation of the 329 tumours was mainly on the lower eyelids (225 tumours), and the medial canthal regions (87 tumours). There was no association between UVR doses at the seven sites of the eyelids and the location of BCCs. The UVR exposure was similar on the upper and lower eyelids, while the number of tumours on the lower eyelids outnumbered the upper lids by a factor of 13 (17 upper, 225 lower)
\end{abstract}

Conclusion-UVR exposure only partially explains the aetiology of periorbital BCC. (Br f Ophthalmol 1998;82:1412-1415) Hospital, Newcastle B L Diffey

Department of
Dermatology,

Gothenborg

University,

Sahlgrenska University

Hospital, Gothenborg,

Sweden

O Larkö

Correspondence to: Gun Lindgren, MD Department of Ophthalmology, Gothenborg University, Sahlgrenska University Hospital, S-413

45 Gothenborg, Sweden.

Accepted for publication 24 June 1998

The incidence of basal cell carcinoma (BCC) is highest in countries with intense sunlight exposure such as in Australia ${ }^{1}$ (823 per 100000 ) and Hawaii $^{2}$ (422 per 100 000). Consequently, solar ultraviolet radiation (UVR) exposure is commonly regarded as an important aetiological factor in the development of this condition, which is the most common form of skin cancer among white people. ${ }^{3}$

The risk of developing BCC has been shown to depend on both the duration and the pattern of UVR exposure. ${ }^{4}$

The increasing incidence of BCC has intensified the search for aetiology, prevention, and improved therapy. In Sweden, with a compara-

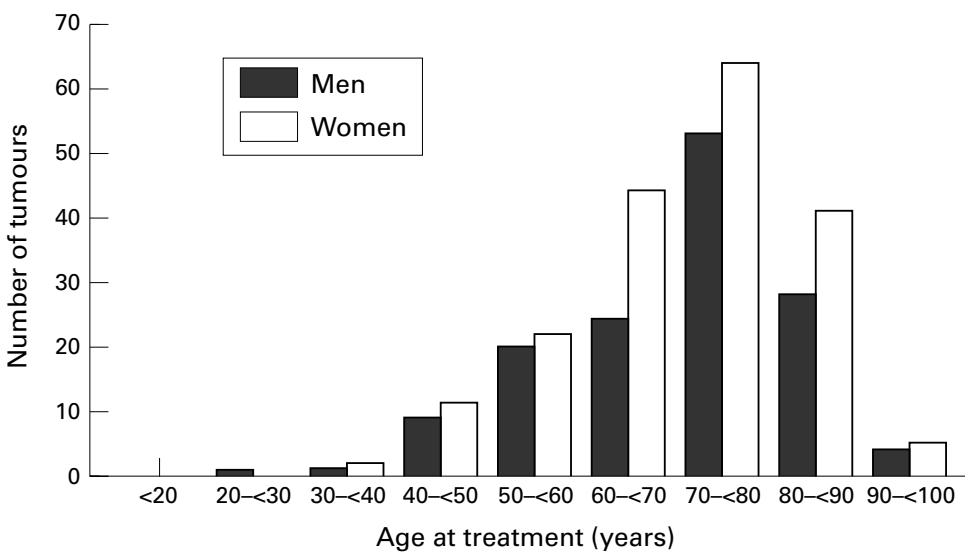

Figure 1 Age and sex distribution of patients treated for eyelid basal cell carcinoma. bly low UVR exposure, the incidence of BCC is now estimated to be at least 15000 cases per year (population nine million). ${ }^{5}$ Most tumours are situated on sun exposed sites of the body, particularly the face. BCCs account for 80$90 \%$ of all malignant tumours of the eyelid.

If UVR is the major aetiological factor for BCC, most tumours should grow on those parts of the eyelid where the UVR exposure is most intense. Attempts to evaluate the UVR dose to the eyelid region have been made by Rosenthal et $a l,{ }^{6}$ who estimated the ocular dose of UVR by placing dosimeters on spectacles. Diffey et $a l^{7}$ measured the UVR exposure at 41 sites of an enlarged model of a head and compared these data with previously published data on the location of BCCs on the face. They found a poor correlation between tumour density and UV dose. The results from the areas measured around the eye were ambiguous.

The purpose of this study was to investigate the relation between the location of the BCCs, and the UVR exposure to specified regions on the eyelids.

\section{Methods}

The location of the BCCs was studied in an outpatient clinic for evaluation and treatment of eyelid tumours (one dermatologist and one ophthalmologist $)^{8}$ in the Sahlgrenska University Hospital, Gothenborg, Sweden.

Histologically verified eyelid BCCs, where the tumour or the $3 \mathrm{~mm}$ safety treatment zone surrounding the tumour affected the eyelid margin or the proximal lacrimal duct, were included in the study. Tumours were assigned to one of seven eyelid regions. To be assigned to the medial part of the eyelids the tumour or the treatment zone should include the punctum. To be assigned to the lateral part of the eyelid the treated part should include the lateral canthus.

In all, 329 tumours in 319 patients were investigated from June 1981 to October 1997. The age (median 73, range 22-98) at treatment and sex distribution (140 men, 189 women) are shown in Figure 1.

The measurements of UVR exposure to the eyelids were performed from May to August 1997. The weather on the measurement days varied from partly cloudy to mainly clear.

The dosimeters were constructed using a skin adhesive tape (Micropore, 3M); one dosimeter with three apertures on each eyelid and one dosimeter with one aperture on each medial canthal area. Apertures were made by a $3 \mathrm{~mm}$ punch and a piece of $50 \mu \mathrm{m}$ thick polysulphone polymer film was attached to the adhesive side covering the apertures. 


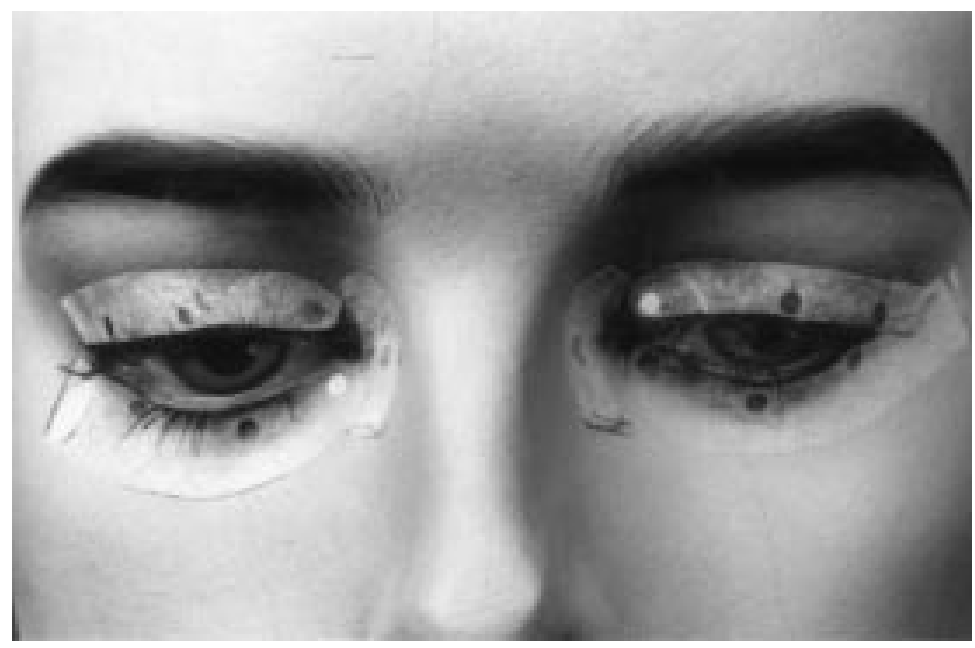

Figure 2 Dosimeters in place on the manikin's eyelids.

In 18 of the exposure measurements we used the head of a female manikin with the dosimeters attached to the eyelids and the apertures close to the eyelid margins (Fig 2). The exposures were made in Gothenborg (latitude $58^{\circ} \mathrm{N}$, altitude $<100$ metres above sea level) with the manikin placed on a unshaded balcony with a dark wooden floor. In 12 of the 18 measurements the manikin was sitting upright and in six the manikin was in recumbent position. The manikin was kept in the same position facing south during the exposure time of $6-8$ hours.

In eight exposures the dosimeters were attached to the skin of the eyelids of one of the two human subjects; person X (female) (Fig 3 $\mathrm{A}$ and $\mathrm{B})$, and person $\mathrm{Y}$ (male). Six exposures took place in Gothenborg and two at $1^{\circ}$ south of Gothenborg. Seven exposures were made on person $\mathrm{X}$ during different conditions; two while sunbathing and walking at the seaside, two while sunbathing and walking in the garden, two while gardening in the sun, and one while sitting in shade. Person $\mathrm{Y}$ was exposed in a recumbent position sunbathing in the garden. The subjects wore the dosimeters for 3-4 hours in the middle of the day.

The optical absorbance at $330 \mathrm{~nm}$ through each aperture was measured. The mean absorbance of unexposed film was subtracted from each of these readings to give the increase in absorbance and this was related to UV
Table 1 Location of 329 eyelid tumours. (The values for left and right eyes are combined)

\begin{tabular}{lll}
\hline & \multicolumn{2}{l}{ Tumours } \\
\cline { 2 - 3 } Location (part of the eyelid) & No & Proportion \\
\hline Upper medial & 4 & 0.012 \\
Upper middle & 10 & 0.030 \\
Upper lateral & 3 & 0.009 \\
Lower medial & $70^{\star}$ & 0.213 \\
Lower middle & $105^{\star}$ & 0.319 \\
Lower lateral & $50^{\star}$ & 0.152 \\
Medial canthal area & 87 & 0.264
\end{tabular}

$\star 21$ of the tumours extended over the entire lower eyelid and were assigned to one of the three sites according to the proportion of the tumours at these three sites.

exposure by an appropriate mathematical expression.

\section{Results}

The distribution of eyelid tumours is listed in Table 1. The tumours were mainly on the lower eyelids (68\%; 225 tumours) and the medial canthal area (26\%; 87 tumours). There was no significant difference between the right and the left eye $(\mathrm{p}=0.44)$.

The measured UVR varied according to exposure time, the position of the head, and weather conditions. Two measurements were excluded from further analysis because of insufficient UV exposure: one with the manikin in recumbent position and one with subject $\mathrm{X}$ in the shade. To compare the relative UVR exposure at each site from the different measurements, the exposure values were converted to the fraction of the mean of the values from all seven sites around each eye for each occasion. The mean and standard deviation of the converted results of the measurements from the dosimeters worn by the manikin and the human subjects are presented in Table 2 . The upper eyelids of the manikin received slightly more UVR than the lower in both sitting and recumbent position. The lateral areas of both the upper and lower eyelids showed low values as well as the medial canthal area. In the human subjects the medial part of both the upper and lower eyelids and the medial canthal areas received the lowest UVR. The lateral part of the eyelids received the highest doses of UVR in the humans.

To calculate the UVR doses of the eyelids and the location of the 329 BCCs located on the eyelids the different areas were regarded to
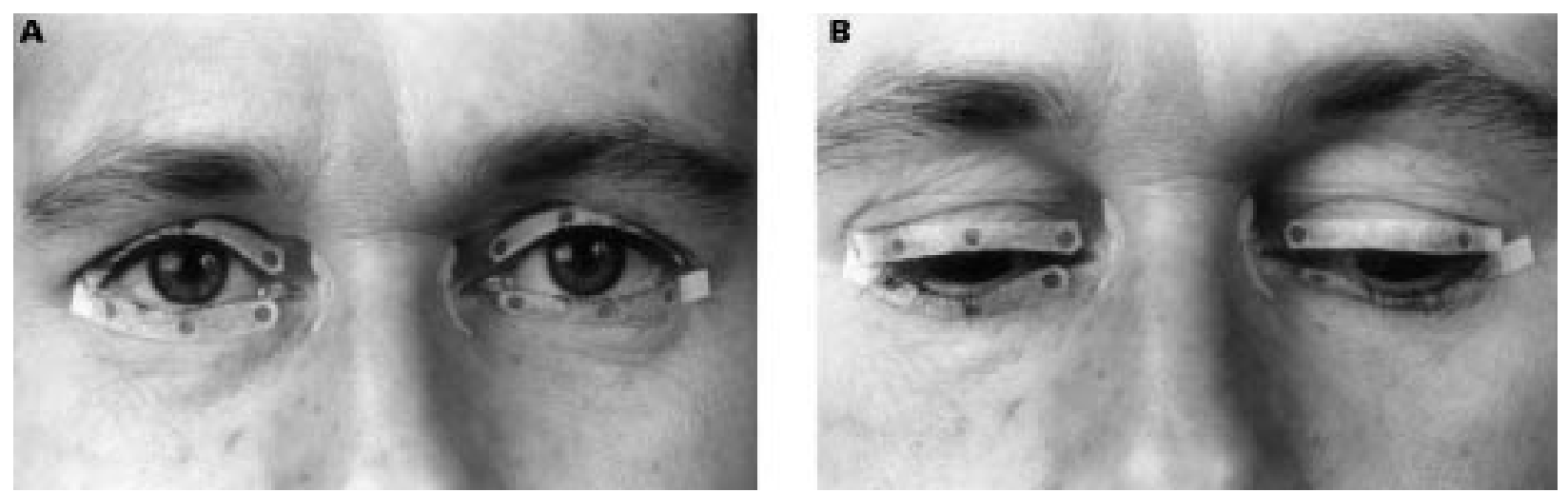

Figure 3 Dosimeters in place in the human subject (A) with open eyes and (B) looking down. 
Table 2 Distribution of ultraviolet radiation to the seven sites around each eye. The read values were converted to fraction of the mean of the readings for each eye from each exposure. The means for the exposures for each site (right and left eyes together) are presented for the manikin in sitting and recumbent position, and for the subjects

\begin{tabular}{llll}
\hline & $\begin{array}{l}\text { Manikin/sitting } \\
\text { Mean of 12 exposures } \\
(S D)\end{array}$ & $\begin{array}{l}\text { Manikin/recumbent } \\
\text { Mean of 5 exposures } \\
(S D)\end{array}$ & $\begin{array}{l}\text { Subjects/various activities } \\
\text { Mean of 7 exposures } \\
(S D)\end{array}$ \\
\hline Upper medial & $1.12(0.23)$ & $1.39(0.31)$ & $0.75(0.22)$ \\
Upper middle & $1.41(0.26)$ & $1.17(0.15)$ & $1.02(0.48)$ \\
Upper lateral & $0.69(0.26)$ & $0.69(0.15)$ & $1.45(0.51)$ \\
Lower medial & $1.14(0.21)$ & $1.07(0.12)$ & $0.79(0.29)$ \\
Lower middle & $1.03(0.21)$ & $1.16(0.17)$ & $1.00(0.38)$ \\
Lower lateral & $0.74(0.20)$ & $0.83(0.13)$ & $1.32(0.41)$ \\
Medial canthal area & $0.87(0.17)$ & $0.70(0.15)$ & $0.69(0.24)$ \\
\hline
\end{tabular}

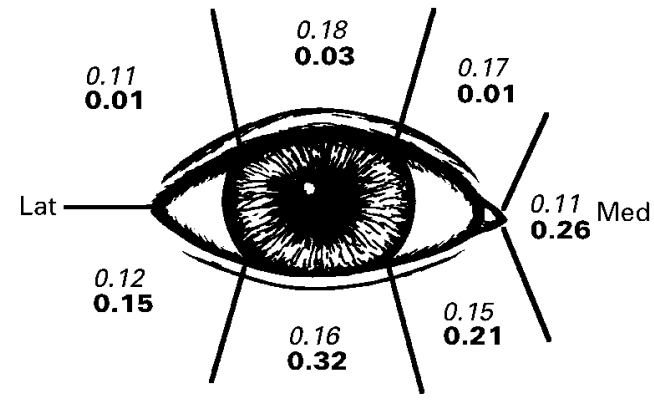

Figure 4 Fraction of the total number of the basal cell carcinomas (bold) and fraction of the total UVR (italics) at the seven different sites of the eyelids (assuming that the areas are approximately of the same size). Right and left eye combined.

be approximately of the same size. Figure 4 shows the tumour distribution and the UVR exposure at the seven sites expressed as a proportion of the total, weighted from all the measurements. BCCs were much more common on the lower eyelid while the UVR exposure did not show preponderance for the lower eyelid. Further, the medial parts of the eyelids received relatively less UV exposure although the incidence of BCCs at this site is high.

\section{Discussion}

The incidence of BCCs increases with age and with no sex predilection. ${ }^{10}$ However, in our study there were more women than men in all age categories except for those younger than 30 years (one young man affected and no women). The higher presence of women in our study is only partly explained by the demographics in our area.

The spectral sensitivity of the polysulphone film is restricted to wavelengths below $330 \mathrm{~nm}$ and consequently measures mainly erythemaproducing UVB. The action spectrum for development of BCC is not known. However, the values at the different sites indicate the UVR exposure at that location and there is no reason to believe that different UV wavelengths should be differently distributed.

Sliney ${ }^{11}$ discussed the difficulties in estimating ocular exposure to UVR. Clearness of the sky, time of the day, time of the year, latitude, altitude, ground reflectance are some of the variables that influence ambient UVR. The fraction of ambient UVR that affects the skin of the eyelids depends, among other things, on attempts to avoid or not avoid sunlight by the choice of outdoor activities, seeking shadow, facing away from the sun, wearing hat, glasses or sunglasses. ${ }^{11}$ In our study the subjects performed different outdoor activities without shading the eyes with a hat or sunglasses. Our study was not aimed at finding UVR dose as a fraction of ambient exposure but to compare the relative UVR exposure at different parts of the eyelids and the medial canthal area. We measured higher UVR doses if the subject was lying down sunbathing than if the subject was walking about in an upright position.

The human subjects as well as the manikin were exposed during the time of the day with the most intense UVR.

In both the manikin head and in the human subjects we found relatively less UVR at the medial canthal area. This can probably be explained by shadowing by the nose. The relative UVR at the different sites of the eyelids of the manikin and the living subjects do not correlate well. The manikin received more UVR in the mid part of the eyelids compared with the subjects who received more UVR in the lateral part of the eyelids. Several factors could account for differences: anatomical differences at the temporal part of the orbit in the humans, the design of the manikin, and the movements of the eyelid in the human subjects.

The human subjects were instructed not to avoid the sun but to move the eyelids as naturally as possible during different activities, keeping the head as much as possible towards the sun. The dosimeters made for the human subjects were as small as possible so as not to disturb the normal movement of the eyelids, such as blinking and squinting. Nevertheless, there were some problems in wearing the dosimeters in bright sunlight for several hours since blinking was slightly hampered. Because of the hot sun and the abnormal blinking, sweat dropped into the eyes causing further irritation. This may have influenced the position of the eyelids and caused more squinting than normally.

Both environmental and hereditary factors are known to increase the risk of developing BCC. There is an increasing risk with age and a fair skin colour. Compared with squamous cell cancer (SCC) the evidence that exposure to sunlight is the predominant cause of BCC is less compelling. SCC occurs almost exclusively on sun exposed skin such as the face, neck, and arms, and the incidence is clearly correlated with geographical latitude, being higher in the more sunny areas of the world. ${ }^{12}$

Even though BCC can occur in skin not exposed to the sun, the evidence is strong that sunlight is an aetiological factor in the development of BCC. In our study the relative UVR did not correspond well with the localisation of the BCCs. In this study as well as in several other studies ${ }^{13-15}$ we found an uneven distribution of the BCCs to the eyelids. The relatively high incidence of BCC in the lower eyelids and the medial canthal area could perhaps be explained by local conditions other than sun exposure. Perhaps the thin epithelium in the medial canthal area allows more UVR to reach the cells of the basal layer. However, the large difference in tumour localisation between the 
upper and lower eyelids is difficult to explain on these grounds.

The lack of association between relative UVR exposure on the eyelids and BCC location indicates that UVR exposure only partially explains the aetiology of periorbital BCC, and there are probably other, yet unidentified, factors that contribute to the development of these tumours.

1 Giles GG, Marks R, Foley P. Incidence of non-melanocytic

skin cancer treated in Australia. BMF 1988;296:13-17.
2 Reizner GT, Chuang T-Y, Elpern DJ, et al. Basal cell carciReizner GT, Chuang T-Y, Elpern DJ, et al. Basal cell carci-
noma in Kauai, Hawaii: the highest documented incidence noma in Kauai, Hawaii: the highest documented incidence
in the United States. 7 Am Acad Dermatol 1993;29:184-9. 3 Marks R. An overview of skin cancers. Cancer 1995;75:607-

12 .

4 Kricker A, Armstrong BK, English DR, et al. A doseresponse curve for sun exposure and basal cell carcinoma. Int F Cancer 1995;60:482-8.

5 Dahl E, Åberg M, Rausing A, et al. Basal cell carcinoma. Cancer 1992;70:104-8.

6 Rosenthal FS, Phoon C, Bakalian AE, et al. The ocular dose of ultraviolet radiation to outdoor workers. Invest Ophthalmol Vis Sci 1988;29:649-56.
7 Diffey BL, Tate TJ, Davis A. Solar dosimetry of the face: the relationship of natural ultraviolet radiation exposure to basal cell carcinoma localisation. Phys Med Biol 1979;24: 931-9.

8 Lindgren G, Larkö O. Long-term follow-up of cryosurgery of basal cell carcinoma of the eyelid. 7 Am Acad Dermatol 1997;36:742-6.

9 Diffey BL. Ultraviolet radiation dosimetry with polysulphone film. In: Diffey BL, ed. Radiation measurement in polysulphone film. London: Academic Press, 1989:135-9.

10 Collin JRO. Basal cell carcinoma in the eyelid region. $B r F$ Ophthalmol 1976;60:806-10.

1 Sliney DH. UV radiation ocular exposure dosimetry. Doc Ophthalmol 1995;88:243-54

12 Kricker A, Armstrong BK, English DR. Sun exposure and non-melanotic skin cancer. Cancer Cause Control 1994;5: 367-92.

13 Doxanas MT, Green WR, Iliff CE. Factors in the successful surgical management of basal cell carcinomas of the eyelids. Am F Ophthalmol 1981;91:726-36.

14 Fraunfelder FT, Zacarian SA, Limmer BL, et al. Cryosurgery for malignancies of the eyelid. Ophthalmology 1980;87: $461-5$.

15 Lederman M. Radiation treatment of cancer of the eyelids. Br F Ophthalmol 1976;60:794-805. 